\title{
陶瓷薄基板材料裂纹预制与断裂韧性评价
}

\author{
马德隆 ${ }^{1,2}$, 包亦望 ${ }^{1,2}$, 万德田 ${ }^{1,2}$, 邱 岩 $^{2}$, 郑德志 ${ }^{2}$, 付 帅 $^{2}$
}

(1. 中国建筑材料科学研究总院有限公司 绿色建筑材料国家重点实验室, 北京 100024; 2. 中国建材检验认证集团 股份有限公司, 北京 100024)

摘 要: 预制长度可控的裂纹以及原位观察裂纹扩展是研究陶瓷薄基板抗断裂行为的两大重点。本研究提出应变诱 导法，通过将基板与黄铜梁粘结形成复合体，利用黄铜梁弯曲变形带动侧面陶瓷薄板受拉侧拉伸变形产生可控裂 纹。在工具显微镜下对复合体进行四点弯曲, 原位观察样品的裂纹扩展情况, 通过调节黄铜梁宽度来控制初始裂纹 长度, 在初始裂纹萌发后继续加载, 使裂纹达到测试断裂韧性的标准长度。将这种测试方法与块体材料断裂韧性的 测试标准进行了对比, 结果表明: 采用该方法预制裂纹后测试断裂韧性具有简易性和可靠性。应变诱导法预制裂纹 成功率高, 裂纹萌发位置及长度可控, 且操作方便, 可推广应用于超薄玻璃等其他超薄脆性材料的断裂韧性评价 和分析裂纹扩展阻力。

关 键 词: 陶瓷薄基板; 预制裂纹; 原位观察; 断裂韧性

中图分类号: TQ174 文献标志码: A

\section{Pre-crack and Fracture Toughness Evaluation of Ceramic Thin Plates}

\author{
MA Delong ${ }^{1,2}$, BAO Yiwang ${ }^{1,2}$, WAN Detian ${ }^{1,2}$, QIU Yan ${ }^{2}$, ZHENG Dezhi $^{2}$, FU Shuai ${ }^{2}$ \\ (1. State Key Laboratory of Green Building Materials, China Building Materials Academy, Beijing 100024, China; 2. China \\ Building Material Test \& Certification Group Co., Ltd., Beijing 100024, China)
}

\begin{abstract}
Controllable length of pre-crack and in-situ observation are two major difficulties in the research of fracture resistance of ceramic materials. In this study, a method of strain-induced cracking was proposed, in which the plate was bonded to a brass beam to form a composite. The bending deformation of brass beam derived the tension deformation in the tension zone of ceramic thin plate to induce the controllable crack. Four-point bending test was conducted under a tool microscope to perform in-situ observation of crack propagation. The initial crack length was controlled by adjusting the width of the tensile zone of the ceramic plate. After the crack initiation, loading continued to make the crack length meet the requirements of fracture toughness test for ceramics. Compared with standard test of fracture toughness of block materials, the above method is simple and reliable. The strain-induced method to pre-crack has high success rate, the position of crack initiation and the length of pre-crack are controllable and easy to operate. It can be widely applied to fracture toughness evaluation and crack propagation resistance analysis of ultra-thin glass and other ultra-thin brittle materials.
\end{abstract}

Key words: ceramic thin plates; pre-crack; in situ observation; fracture toughness

收稿日期：2020-11-02; 收到修改稿日期：2020-11-27; 网络出版日期：2021-12-10

基金项目: 国家重点研发计划重大科学仪器设备开发专项(2018YFF01012404); 国家自然科学基金重点项目(52032011) National Key R\&D Program of China (2018YFF01012404); National Natural Science Foundation of China (52032011)

作者简介：马德隆(1993-), 男, 博士研究生. E-mail: madelong@ctc.ac.cn MA Delong(1993-), male, PhD candidate. E-mail: madelong@ctc.ac.cn

通信作者：包亦望，教授. E-mail: baoyw@ctc.ac.cn BAO Yiwang, professor. E-mail: baoyw@ctc.ac.cn 
陶瓷薄基板因具有介电常数小、高频特性好、 绝缘性能好和热失配率低等优异性能而广泛应用于 半导体、航空航天和国防军工等领域的产品封装 ${ }^{[1-3]}$ 。 陶瓷薄基板在应用中常常伴随着颠簸震动等复杂力 学环境, 同时由于使用时的反复热循环过程, 导致 基板受到较大残余应力的影响, 因而对材料的力学 性能有着较高的要求 ${ }^{[4-5]}$ 。断裂韧性作为陶瓷材料最 为重要的本征力学性能之一, 是反映材料阻止裂纹 扩展的能力。准确测试陶瓷薄基板的断裂韧性, 评 估材料的裂纹扩展过程, 对保障构件的服役可靠性 和安全应用具有重要意义 ${ }^{[2,5-7]}$ 。

目前, 陶瓷薄基板断裂韧性通常采用同批材料随 炉样品的断裂韧性作为参考, 缺乏直接的测试途径, 造成这一情况的主要原因之一在于大部分陶瓷薄基 板厚度较薄, 难以加工成标准样品进行直接测试。

国内外的研究表明, 通过预制适当长度的初始 裂纹再进行测试, 可准确评价材料的断裂韧性 ${ }^{[8-10]}$ 。 然而, 由于陶瓷薄基板的低韧性和高弹性模量, 在 预裂时很难控制裂纹的萌生和止裂，特别是直裂 纹。对于大多数脆性材料, 宏观裂纹一旦在静载荷 下萌生, 就会迅速扩展。因此, 在预裂过程中的陶瓷 样品往往表现出灾难性的断裂或不开裂 ${ }^{[8]}$ 。因此, 预 制长度可控的裂纹以及原位观察裂纹扩展情况成为 制约陶瓷薄基板抗断裂行为研究的两大难点。

Miyazaki 等 ${ }^{[2,5]}$ 提出改进的单边预裂板法以及 单边 $\mathrm{V}$ 型切口板法, 并基于这两种方法制订了国际 标准 ISO $21113^{[11]}$ 。单边预裂板法采用三点弯曲并 结合声发射装置进行基板裂纹预制，然而部分陶瓷 断裂时的声信号不易捕捉，同时该方法预制裂纹的 长度无法控制, 导致预制成功率较低, 且三点弯曲 方式不易控制裂纹扩展方向。单边 $\mathrm{V}$ 型切口板法采 用刀片加工 $\mathrm{V}$ 型切口, 该方法制备的 $\mathrm{V}$ 形缺口根部 没有形成尖锐裂纹，所测得的断裂韧性值误差较大。 同时，上述两种方法均无法实现裂纹的原位观察。 An 等 ${ }^{[12-13]}$ 对 CVD 类金刚石薄膜预制裂纹并通过增 加基体厚度的方式提高了预制裂纹的成功率。

本实验室曾提出应变控制法 ${ }^{[8]}$, 采用斜切口梁 样品在显微镜下水平加载, 观察到脆性材料裂纹的 缓慢扩展过程，控制了裂纹长度，相关方法的原理 和装置如图 1 所示。然而, 由于陶瓷基板厚度较薄, 无法在一端制作斜切口。因此还需探索新方法实现 厚度较薄材料的裂纹观察。

\section{1 基本原理}

本研究提出应变诱导法, 将陶瓷薄基板与弹性

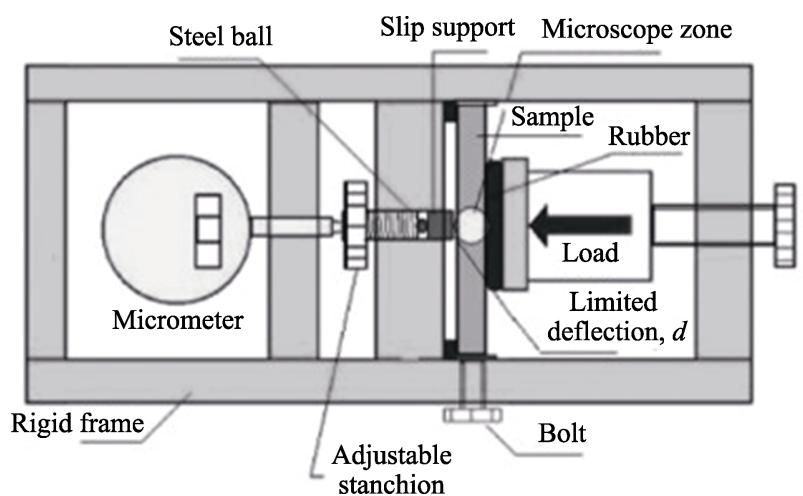

图 1 应变控制法预制裂纹示意图 ${ }^{[8]}$

Fig. 1 Schematic diagram of pre-cracking in strain control method

模量较低的黄铜梁粘接形成复合体样品，在工具显 微镜下进行水平方向四点弯曲加载, 实现样品裂纹 预制与原位观察。裂纹萌发是由于加载过程中黄铜 梁变形带动基板发生变形, 从而诱导基板产生裂 纹。同时, 黄铜梁还可以抑制基板发生失稳扩展, 避 免产生灾难性断裂。在此基础上，通过在样品表面 预制缺陷，可控制初始裂纹萌发位置。通过调节样 品受拉区宽度, 控制初始裂纹长度。在初始裂纹萌 发后继续加载, 使得裂纹继续扩展, 直至达到符合 断裂韧性测试要求。

\section{1 初始裂纹萌发位置与长度控制}

在陶瓷薄基板上直接进行裂纹预制，会由于裂 纹的失稳扩展出现灾难性断裂, 预制成功率较低, 故将陶瓷薄基板加工成长条状，与弹性模量较小的 黄铜梁粘接形成复合体样品, 如图 2 所示。

对样品进行弯曲加载, 使陶瓷薄基板样品的下 部受拉，上部受压，当受拉区产生裂纹并扩展到受 压区时裂纹受阻停止扩展，从而形成了一条通透裂 纹。由于陶瓷薄基板样品受拉区的裂纹扩展有一定 的惯性，因此通过调整黄铜梁宽度，调整基板的中 性轴位置, 可以减小基板的受拉区宽度 $B_{\mathrm{t}}$, 如图 3 所示。

另外，在预制裂纹前，利用金刚石切割机在样

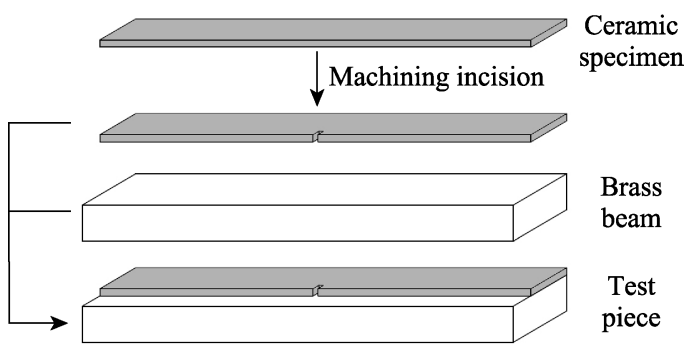

图 2 测试样品组成示意图

Fig. 2 Schematic diagram of test piece composition 


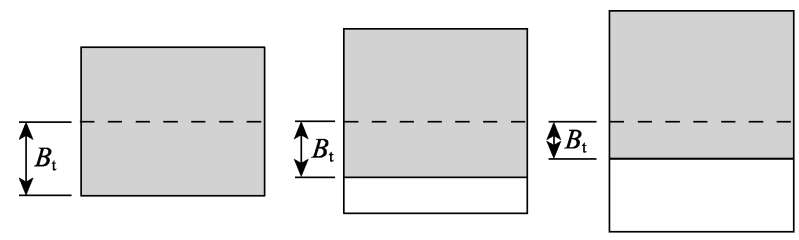

图 3 受拉区宽度随黄铜梁宽度增加而减小

Fig. 3 Width of tensile zone decreases with increase of brass beam width

品的长边中心边缘位置加工微小切口, 确保裂纹萌 发位置位于陶瓷薄基板的中心。

\section{2 裂纹原位观察与长度控制扩展}

设计一套可用于水平方向测试四点弯曲的夹具, 如图 4 所示, 将夹具放置在工具显微镜下进行陶瓷 预裂纹试验。以非常缓慢的加载速度施加水平载荷, 利用工具显微镜原位观察陶瓷薄样品初始裂纹从萌 发到逐渐扩展的过程。在样品的初始裂纹萌生后, 继续缓慢加载, 使得裂纹持续扩展, 直至符合断裂 㓞性测试的标准长度, 即陶瓷薄基板宽度的 0.35 0.60 倍 $^{[14]}$ 。

\section{2 实验方法}

\section{1 制备样品}

选用氧化铝、碳化硅和氮化硅三种陶瓷薄基板 材料, 尺寸为 $50.0 \mathrm{~mm} \times 50.0 \mathrm{~mm} \times 0.6 \mathrm{~mm}$, 基本力学 性能如表 1 所示。

利用金刚石切割机将陶瓷薄基板切割成 $36 \mathrm{~mm} \times$ $4 \mathrm{~mm} \times 0.6 \mathrm{~mm}$ 宽的样品。在样品中央边缘加工切口, 用 502 胶粘接陶瓷薄基板和黄铜梁形成测试样品, 如图 5 所示，黄铜梁的尺寸分别选用 $36 \mathrm{~mm} \times(4,5$, $5.5,6.5) \mathrm{mm} \times 3 \mathrm{~mm} 4$ 种规格。

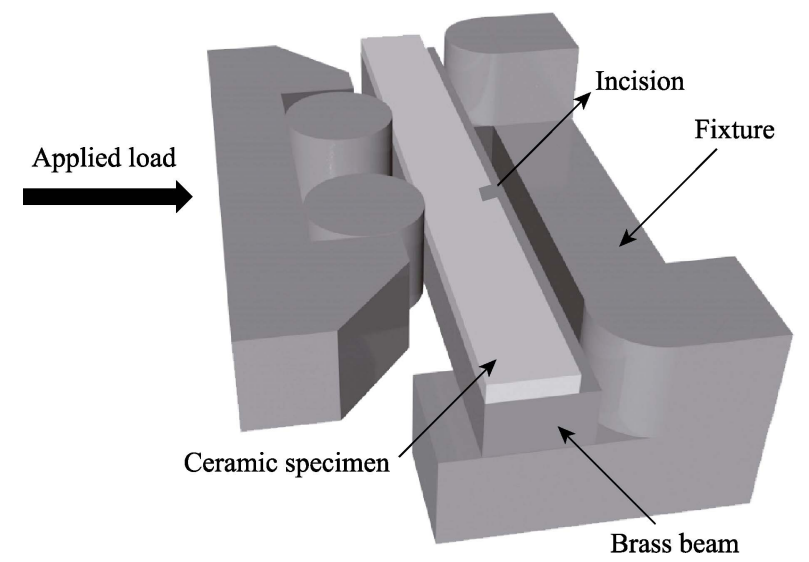

图 4 水平加载四点弯曲预裂纹示意图

Fig. 4 Schematic diagram of pre-cracking by four-point bending test in horizontal direction
表 1 陶瓷薄基板的基本力学性能

Table 1 Basic mechanical properties of three types of ceramic thin plates

\begin{tabular}{cccc}
\hline Material & $\begin{array}{c}\text { Elastic } \\
\text { modulus/GPa }\end{array}$ & $\begin{array}{c}\text { Bending } \\
\text { strength/MPa }\end{array}$ & $\begin{array}{c}\text { Vickers } \\
\text { hardness/GPa }\end{array}$ \\
\hline $\mathrm{Al}_{2} \mathrm{O}_{3}$ & $(305 \pm 10)$ & $(315 \pm 20)$ & $(19.8 \pm 0.1)$ \\
$\mathrm{SiC}$ & $(360 \pm 12)$ & $(340 \pm 28)$ & $(20.1 \pm 0.3)$ \\
$\mathrm{Si}_{3} \mathrm{~N}_{4}$ & $(280 \pm 8)$ & $(425 \pm 33)$ & $(15.9 \pm 0.6)$ \\
\hline & & (b) & \\
(a) & & & \\
& & & \\
& & & \\
& & $1 \mathrm{~mm}$ &
\end{tabular}

图 5 陶瓷薄基板与黄铜梁粘结形成复合体

Fig. 5 Microscopic images of composite bonded by ceramic thin plate and brass beam

(a) Machining incision in the edge of ceramic thin plate specimen; (b) Composite bonded by ceramic thin plate and brass beam bonded to form a composite

\section{2 预制裂纹和原位观察}

将预制好缺陷的复合体放置于夹具中进行四点 弯曲测试, 利用手摇式加载装置缓慢施加载荷, 在 工具显微镜下观察样品裂纹萌发及扩展过程, 如图 6 所示。

\section{3 测试断裂韧性}

用二氯甲烷浸泡将复合体样品的陶瓷薄基板和 黄铜梁分离。采用三点弯曲法测试陶瓷薄基板断裂 韧性; 采用带有垂直狭缝的夹具测试三点弯曲强 度, 如图 7 所示, 狭缝的宽度比试样的厚度大 0.02 $0.03 \mathrm{~mm}$ 。另外, 采用国家标准 GB/T 23806 规定的 单边预裂纹梁 ${ }^{[14]}$, 测试相同工艺制备的块体材料的 断裂韧性。

将陶瓷薄基板插入狭缝中, 使样品在测试过程 中保持坚直, 再以 $0.5 \mathrm{~mm} / \mathrm{min}$ 的加载速度施加压缩 载荷, 直至样品断裂。

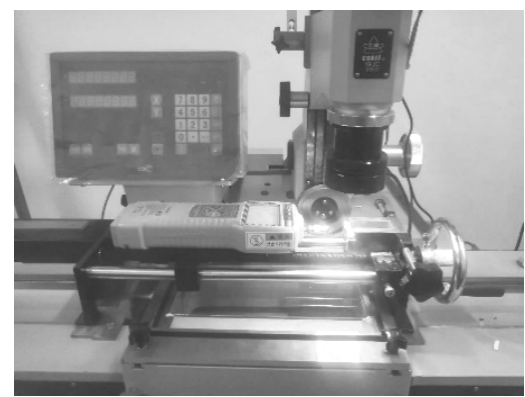

图 6 工具显微镜下四点弯曲法预制裂纹

Fig. 6 Diagram of pre-cracking by four-point bending test under tool microscope 


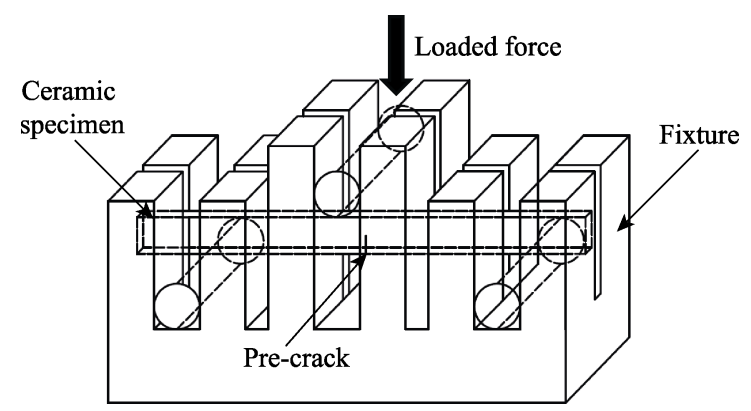

图 7 采用三点弯曲法测试断裂韧性示意图

Fig. 7 Schematic diagram of three-point bending test of fracture toughness

样品的断裂韧性 $K_{\mathrm{IC}}$ ，用式(1)进行计算：

$$
\begin{aligned}
K_{\mathrm{IC}}= & \left(\frac{P \cdot S}{B \cdot W^{3 / 2}}\right)\left\{\frac{3}{2} \cdot\left(\frac{a}{W}\right)^{1 / 2} \cdot Y\left(\frac{a}{W}\right)\right\} \\
Y\left(\frac{a}{W}\right)= & 1.964-2.837\left(\frac{a}{W}\right)+13.711\left(\frac{a}{W}\right)^{2}- \\
& 23.250\left(\frac{a}{W}\right)^{3}+24.129\left(\frac{a}{W}\right)^{4}
\end{aligned}
$$

式中, $P$ 为样品断裂时的最大载荷, $S$ 为三点弯曲试 验的跨距, $B$ 为样品的厚度, $W$ 为样品的宽度, $a$ 为预 制裂纹的长度。

\section{3 结果与讨论}

\section{1 裂纹扩展过程}

氧化铝陶瓷薄基板试样初始裂纹的萌生和扩展 过程如图 8 所示。随着载荷的逐渐增大, 样品开始 产生挠度变形。与陶瓷薄基板相比, 黄铜梁的弹性 模量较小, 在相同载荷作用下, 黄铜梁的挠度变形 远大于陶瓷的挠度变形。利用黄铜梁弯曲变形来诱 导陶瓷薄样品受拉部分发生开裂, 产生应变诱导裂 纹。从图 8 中可以看出, 在逐渐加载达到一定程度 时, 基板会突然萌发一条初始裂纹(图 8(a)), 之后继 续缓慢加载直至裂纹扩展至断裂韧性测试要求的尺 寸(图 8(b)), 表明四点弯曲法可在基板表面预制长 度可控的裂纹, 并且制备的裂纹尖端与样品水平方 向近乎于垂直，如图 8(c)所示。

采用三点弯曲法预制裂纹时, 要求微缺陷与上 压头位置完全在载荷中轴线上, 若稍有偏移则产生 的裂纹将会出现倾斜的情况, 如图 9 所示。造成这 一情况的原因在于：当微缺陷位置与上压头位置有 偏移时, 裂纹扩展将朝上压头方向倾斜; 而四点弯 曲方式在两个上压头之间的区域内样品受到的弯矩 为定值, 在此区域内样品受到的拉应力相同, 促使

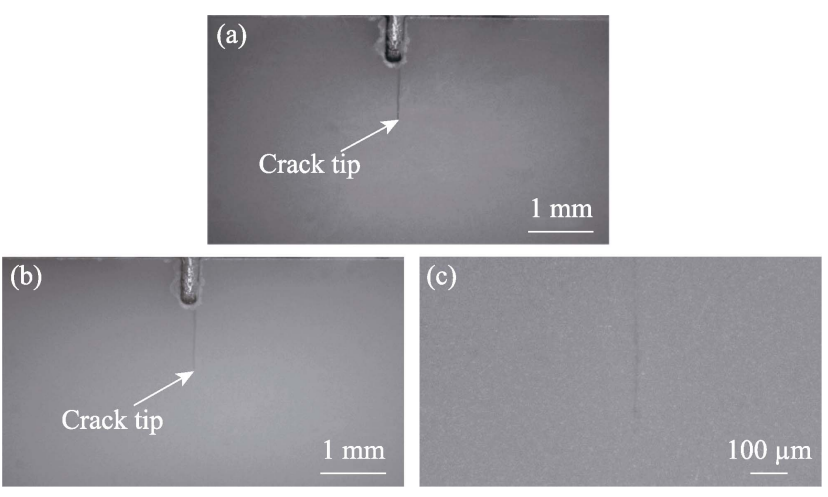

图 8 采用四点弯曲法预制氧化铝基板裂纹及扩展过程

Fig. 8 In- situ observation of crack initiation and extension in $\mathrm{Al}_{2} \mathrm{O}_{3}$ plate

(a) Initial crack; (b) Continue loading until the crack meet the requirements of fracture toughness test; (c) Crack tip

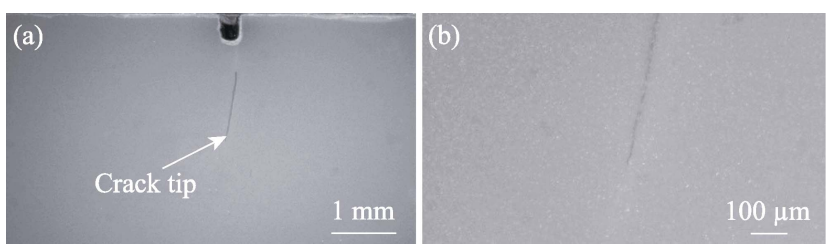

图 9 采用三点弯曲法预制的裂纹

Fig. 9 Pre-crack by three-point bending test (a) Pre-crack; (b) Crack tip

从缺陷位置开始的裂纹沿垂直方向开裂。

采用四点弯曲方法预裂裂纹的优势为：预制裂 纹成功率高, 裂纹萌发位置及长度可控, 且操作方 便; 利用光学显微镜可以在样品表面原位监测裂纹 的扩展行为, 为研究陶瓷薄基板中阻止宏观裂纹的 提供了思路。

\section{2 不同宽度黄铜梁预制裂纹}

实验表明，当陶瓷片与金属梁样品的弯曲中性 轴重合时, 预制的裂纹长度总是超过样品宽度的一 半。为控制裂纹长度达到宽度的 $0.35 \sim 0.60$ 倍, 通过 增加黄铜梁宽度使陶瓷薄基板样品中性轴偏移到黄 铜梁的受压区，从而减小样品受拉区宽度，进而缩 短样品表面初始裂纹的长度。采用不同宽度黄铜梁 预制的初始裂纹长度如图 10 所示。当黄铜梁宽度过 大时, 会因粘结剂强度不足出现陶瓷薄基板与样品 脱粘的现象，降低预制裂纹的成功率。因此，对于陶 瓷薄基板宽度为 $4 \mathrm{~mm}$ 的样品, 推荐的黄铜梁宽度 为 $5 \sim 6 \mathrm{~mm}$ 。

\section{3 测试预制裂纹样品的断裂韧性}

经预制裂纹后三种陶瓷薄基板断裂韧性测试结 果如表 2 所示。从表中可以看出，陶瓷薄基板与同 种工艺制备的块体材料断裂韧性的测试结果基本一 致，证明采用应变诱导法预制裂纹后的断裂韧性测 试结果是可靠的。 


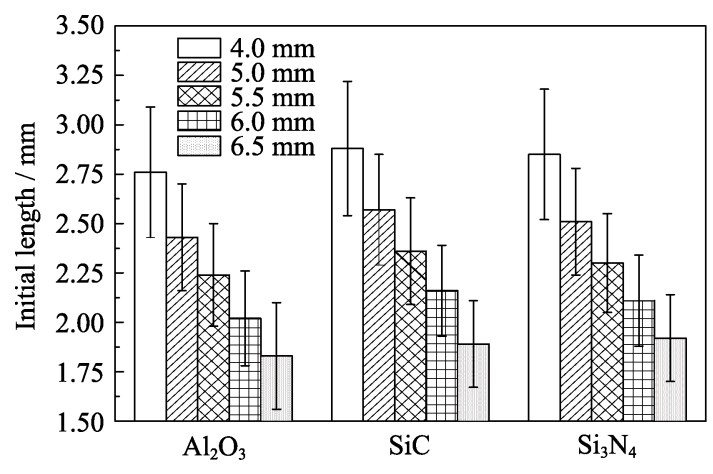

图 10 不同宽度黄铜梁预制裂纹的初始长度

Fig. 10 Pre-crack initial length by using brass beam of different widths

表 2 陶瓷薄基板与陶瓷块体断裂韧性对比

Table 2 Fracture toughness of ceramic thin plate and ceramic block

\begin{tabular}{ccccc}
\hline $\begin{array}{c}\text { Material } \\
\text { type }\end{array}$ & $\begin{array}{c}\text { Thickness } \\
\text { of the } \\
\text { specimen/mm }\end{array}$ & $\begin{array}{c}\text { Number of } \\
\text { specimen }\end{array}$ & $\begin{array}{c}\text { Number of } \\
\text { successful } \\
\text { pre-cracks }\end{array}$ & $\begin{array}{c}\text { Fracture } \\
\text { toughness/ } \\
\left(\mathrm{MPa} \cdot \mathrm{m}^{1 / 2}\right)\end{array}$ \\
\hline $\mathrm{Al}_{2} \mathrm{O}_{3}$ & 0.6 & 15 & 15 & $(4.17 \pm 0.26)$ \\
& 3 & 10 & 8 & $(4.21 \pm 0.25)$ \\
$\mathrm{SiC}$ & 0.6 & 15 & 14 & $(3.81 \pm 0.24)$ \\
& 3 & 10 & 7 & $(3.72 \pm 0.22)$ \\
$\mathrm{Si}_{3} \mathrm{~N}_{4}$ & 0.6 & 15 & 14 & $(5.28 \pm 0.31)$ \\
& 3 & 10 & 8 & $(5.36 \pm 0.33)$ \\
\hline
\end{tabular}

\section{4 结论}

1)提出陶瓷薄基板裂纹预制的应变诱导法，通 过控制裂纹尖端的应力状态实现了预制陶瓷材料长 度可控的裂纹以及原位观察裂纹扩展，为研究陶瓷 薄基板的断裂行为提供了依据。

2)与三点弯曲法相比, 采用四点弯曲法预制裂 纹, 可避免因微缺陷位置与压头不对应导致的由裂 纹倾斜产生的预制失败。

3)在陶瓷与黄铜梁的受压面保持平齐的条件下， 陶瓷薄基板样品受拉区宽度随黄铜梁宽度的增大而 逐渐减小, 使形成的初始裂纹长度随之减小, 裂纹 通常止裂于金属梁中性轴边的受压侧。

4)与同种工艺制备的块体陶瓷材料断裂韧性相
比，采用应变诱导法测试预制裂纹样品的断裂韧性 稳定可靠。

5)应变诱导法预制裂纹平均成功率为 90\% 95\%, 裂纹萌发位置及长度可控，操作方便，可推广应用 于超薄玻璃等其他超薄脆性材料的预制裂纹和裂纹 阻力分析。

\section{参考文献:}

[1] KHAZAKA R, MENDIZABAL L, HENRY D, et al. Survey of hightemperature reliability of power electronics packaging components. IEEE Trans. Power Electron., 2015, 30(5): 2456-2464.

[2] MIYAZAKI H, YOSHIZAWA Y, HIRAO K, et al. Measurements of fracture toughness of ceramic thin plates through single-edge V-notch plate method. J. Eur. Ceram. Soc., 2016, 36(16): 4327-4331.

[3] 曾小亮, 孙蓉, 于淑会, 等. 电子封装基板材料研究进展及发展 趋势. 集成技术, 2014, 3(6): 76-83.

[4] SMIRNOV S V, ROMANOV B P, STRELOV K K. The failure of thin ceramic plates under intensive heat flows. Ceram. Int., 1991, 17(3): 205-206.

[5] MIYAZAKI H, YOSHIZAWA Y I , HIRAO K, et al. Evaluation of fracture toughness of ceramic thin plates through modified single edge-precracked plate method. Scr. Mater., 2015, 103: 34-36.

[6] STROBL S, RASCHE S, KRAUTGASSER C, et al. Fracture toughness testing of small ceramic discs and plates. J. Eur. Ceram. Soc., 2014, 34(6): 1637-1642.

[7] MIYAZAKI H, YOSHIZAWA Y I, HIRAO K, et al. Round-robin test on the fracture toughness of ceramic thin plates through modified single edge-precracked plate method. J. Eur. Ceram. Soc., 2016, 36(13): 3245-3248.

[8] BAO Y W, ZHOU Y C. A new method for precracking beam for fracture toughness experiments. J. Am. Ceram. Soc., 2010, 89(3): 1118-1121.

[9] SAKAI M, BRADT R C. Fracture toughness testing of brittle materials. Metall. Rev., 1993, 38(2): 53-78.

[10] 万德田, 魏永金, 包亦望, 等. 陶瓷断裂韧性测试方法准确性和 简便性比较分析. 硅酸盐学报, 2019, 47(8): 1080-1088.

[11] ISO 21113-2018, Fine ceramics (advanced ceramics, advanced technical ceramics)-Test method for fracture toughness of monolithic ceramic thin plates at room temperature.

[12] AN K, CHEN L X, YAN X B, et al. Fracture strength and toughness of chemical-vapor-deposited polycrystalline diamond films. Ceram. Int., 2018, 44(15): 17845-17851.

[13] LU F X, JIANG Z, TANG W Z, et al. Accurate measurement of strength and fracture toughness for miniature-size thick diamondfilm samples by three-point bending at constant loading rate. Diamond Relat. Mater., 2001, 10(3-7): 770-774.

[14] GB/T 23806-2009, 精细陶瓷断裂㓞性试验方法单边预裂纹梁 (SEPB)法. 